Pacific Journal of Mathematics

COMPACT PERTURBATIONS OF ORTHOGONAL 


\section{COMPACT PERTURBATIONS OF ORTHOGONAL POLYNOMIALS}

\section{Paul Nevai and Walter Van Assche}

We investigate orthogonal polynomials on the real line defined by a recurrence relation for which the recurrence coefficients behave asymptotically like a given system of recurrence coefficients. We give the asymptotic behavior of the orthogonal polynomials (relative to the given comparison system of orthogonal polynomials) and from this we deduce properties of the orthogonality measure.

1. Introduction. Let $\mu_{0}$ be a positive unit measure on the real line such that its support contains infinitely many points and all the moments are finite. Assume moreover that the Hamburger moment problem is determined. Denote by $p_{n}\left(x ; \mu_{0}\right)(n=0,1,2, \ldots)$ the normalized orthogonal polynomials for the measure $\mu_{0}$, i.e.

$$
\begin{gathered}
\int p_{n}\left(x ; \mu_{0}\right) p_{m}\left(x ; \mu_{0}\right) d \mu_{0}(x)=\delta_{m, n}, \quad m, n>0, \\
p_{n}\left(x ; \mu_{0}\right)=\gamma_{n}^{0} x^{n}+\cdots, \quad \gamma_{n}^{0}>0 .
\end{gathered}
$$

Let $a_{n}^{0}>0(n=1,2, \ldots)$ and $b_{n}^{0}(n=0,1,2, \ldots)$ be the recurrence coefficients for these orthogonal polynomials, so that

$$
\begin{aligned}
x p_{n}\left(x ; \mu_{0}\right)= & a_{n+1}^{0} p_{n+1}\left(x ; \mu_{0}\right)+b_{n}^{0} p_{n}\left(x ; \mu_{0}\right) \\
& +a_{n}^{0} p_{n-1}\left(x ; \mu_{0}\right), \quad n \geq 0,
\end{aligned}
$$

with initial values $p_{-1}\left(x ; \mu_{0}\right)=0$ and $p_{0}\left(x ; \mu_{0}\right)=1$. We will study a new sequence of orthogonal polynomials $p_{n}(x ; \mu)(n=0,1,2, \ldots)$ with recurrence coefficients $a_{n}(n=1,2, \ldots)$ and $b_{n}(n=0,1,2$, ...) such that

$$
\lim _{n \rightarrow \infty}\left|a_{n}-a_{n}^{0}\right|=0, \quad \lim _{n \rightarrow \infty}\left|b_{n}-b_{n}^{0}\right|=0 .
$$

The orthogonal polynomials $p_{n}(x ; \mu)(n=0,1,2, \ldots)$ are said to be a compact perturbation of the comparison system $p_{n}\left(x ; \mu_{0}\right)$ $(n=0,1,2, \ldots)$ because the corresponding Jacobi matrix $J$ with entries $b_{n}$ on the diagonal and $a_{n}$ on the subdiagonals is a compact perturbation of the Jacobi matrix $J_{0}$ with entries $b_{n}^{0}$ and $a_{n}^{0}$. As a special case one may consider finite perturbations (of order $m \in \mathbb{Z}^{+}$) 
$p_{n}\left(x ; \mu_{m}\right) \quad(n=0,1,2, \ldots)$ for which the recurrence coefficients satisfy

$$
a_{n+1}=a_{n+1}^{0}, \quad b_{n}=b_{n}^{0}, \quad n \geq m .
$$

Notice that for $m=0$ we get the comparison system itself. One of the most relevant questions is to find a closed expression for the orthogonality measure $\mu_{m}$ for these orthogonal polynomials and the weak limit as $m \rightarrow \infty$. This problem has been treated for some specific comparison systems: if $p_{n}\left(x ; \mu_{0}\right)=U_{n}(x)$, the Chebyshev polynomials of the second kind, then $a_{n+1}^{0}=1 / 2$ and $b_{n}^{0}=0$ for $n \geq 0$, and a detailed study has been done in [21]. A scattering method was used by Geronimo and Case [11] for finite perturbations of the Chebyshev polynomials of the second kind and there are explicit formulas in [9]. These authors also have results when $m$ tends to infinity. For a survey of these results we refer to [28]. Geronimo et al. [12] treat the case where the recurrence coefficients of the comparison system are periodic with period $N>1$. Geronimus [14] was one of the first authors to give a detailed account of finite perturbations of Chebyshev polynomials of the second kind and of orthogonal polynomials with asymptotically periodic recurrence coefficients. See also Grosjean [15], [16] for such finite perturbations.

In what follows we will frequently use associated polynomials of order $k \in \mathbb{Z}^{+}$. These polynomials $p_{n}^{(k)}\left(x ; \mu_{0}\right)(n=0,1,2, \ldots)$ are defined by the shifted recurrence relation

$$
\begin{aligned}
x p_{n}^{(k)}\left(x ; \mu_{0}\right)= & a_{n+k+1}^{0} p_{n+1}^{(k)}\left(x ; \mu_{0}\right)+b_{n+k}^{0} p_{n}^{(k)}\left(x ; \mu_{0}\right) \\
& +a_{n+k}^{0} p_{n-1}^{(k)}\left(x ; \mu_{0}\right), \quad n \geq 0,
\end{aligned}
$$

with initial values $p_{-1}^{(k)}\left(x ; \mu_{0}\right)=0$ and $p_{0}^{(k)}\left(x ; \mu_{0}\right)=1$. For $k=0$ these associated polynomials reduce to the comparison system and we therefore omit the superscript for $k=0$. One can easily verify that

$$
p_{n-k}^{(k)}\left(x ; \mu_{0}\right)=a_{k}^{0} \int \frac{p_{n}\left(x ; \mu_{0}\right)-p_{n}\left(t ; \mu_{0}\right)}{x-t} p_{k-1}\left(t ; \mu_{0}\right) d \mu_{0}(t) .
$$

It is important to realize that $p_{n-k}^{(k)}\left(x ; \mu_{0}\right)$ is, for every $k \in \mathbb{Z}^{+}$, a solution of the recurrence relation (1.1) but with initial conditions $p_{-1}^{(k)}\left(x ; \mu_{0}\right)=0$ and $p_{0}^{(k)}\left(x ; \mu_{0}\right)=1$. We will also need the functions of the second kind $q_{n}\left(x ; \mu_{0}\right)(n=0,1,2, \ldots)$ defined as

$$
q_{n}\left(x ; \mu_{0}\right)=\int \frac{p_{n}\left(t ; \mu_{0}\right)}{x-t} d \mu_{0}(t), \quad x \in \mathbb{C} \backslash \operatorname{supp}\left(\mu_{0}\right) .
$$


A straightforward analysis reveals that these functions of the second kind are also a solution of the recurrence relation (1.1), but with initial conditions $a_{0}^{0} q_{-1}\left(x ; \mu_{0}\right)=1$ and $q_{0}\left(x ; \mu_{0}\right)=\int \frac{1}{x-t} d \mu_{0}(t)$. This sequence is important because this is, for $x \in \mathbb{C} \backslash \operatorname{supp}\left(\mu_{0}\right)$, the minimal solution of the recurrence relation (1.1). Observe that $q_{0}\left(x ; \mu_{0}\right)$ is the Stieltjes transform of the measure $\mu_{0}$. If one knows $q_{0}\left(x ; \mu_{0}\right)$ for $x \in \mathbb{C} \backslash \mathbb{R}$, then one also knows the measure $\mu_{0}$ by Stieltjes' inversion formula (Wintner [30, pp. 93-96], [27, p. 175]):

$$
\begin{aligned}
& \frac{1}{2} \mu_{0}(\{x\})+\frac{1}{2} \mu_{0}(\{y\})+\mu_{0}(] x, y[) \\
& \quad=\lim _{\varepsilon \rightarrow 0+} \frac{1}{2 \pi i} \int_{x}^{y}\left\{q_{0}\left(t-i \varepsilon ; \mu_{0}\right)-q_{0}\left(t+i \varepsilon ; \mu_{0}\right)\right\} d t .
\end{aligned}
$$

Associated polynomials and functions of the second kind already appear in Stieltjes' fundamental work [25]. Some interesting properties and formulas may be found in the works of Perron [23] and Geronimus [14]. Functions of the second kind and associated polynomials are usually only studied for classical orthogonal polynomials (Askey and Wimp [2], Barrucand and Dickinson [5], Chihara [7], Grosjean [17], Sherman [24], Szegö [26] and Wimp [29]) but recently more general orthogonal polynomials have also been considered ([4], [6], [10], [22]). If the measure $\mu_{0}$ satisfies some extra regularity conditions, then the functions of the second kind may be extended to the support of $\mu_{0}$ and then there is a simple relationship with the measure $\mu_{0}$ and the function $q_{n}\left(x ; \mu_{0}\right)$, which is essentially given by Privalov's theorem [31, §7.4]:

LEMMA 1. Suppose $\mu_{0}$ is an absolutely continuous measure on the interval $[a, b]$ ( $a$ and $b$ finite), with weight function $w_{0}(x)$. If $w_{0}$ is of Dini type on $[a, b]$, i.e.

$$
\int_{0}^{b-a} \frac{\omega\left(w_{0} ; t\right)}{t} d t<\infty,
$$

where $\omega\left(w_{0} ; t\right)$ is the modulus of continuity of $w_{0}$ on $[a, b]$, defined by

$$
\omega\left(w_{0} ; t\right)=\sup \left\{\left|w_{0}(x)-w_{0}(y)\right|: x, y \in[a, b],|x-y|<t\right\},
$$

then the Cauchy principal value integral

$$
f_{a}^{b} \frac{f(t) w_{0}(t)}{x-t} d t
$$


exists for every $x \in] a, b[$ and every polynomial $f$ and

$$
\begin{aligned}
q_{n}\left(x ; \mu_{0}\right) & =\lim _{\varepsilon \rightarrow 0+} q_{n}\left(x+i \varepsilon ; \mu_{0}\right) \\
& =\int_{a}^{b} \frac{p_{n}\left(t ; \mu_{0}\right) w_{0}(t)}{x-t} d t-i \pi p_{n}\left(x ; \mu_{0}\right) w_{0}(x), \\
& a<x<b .
\end{aligned}
$$

2. The comparison equation. It is very natural to compare $p_{n}(x ; \mu)$ with $p_{n}\left(x ; \mu_{0}\right)$ because the recurrence coefficients of both systems are asymptotically equal. The general setup for such a comparison is given by Geronimo et al. [12, Eq. III.8] (see also [27, p. 54]). Define

$$
\hat{p}_{n}(x ; \mu)=\left(\prod_{j=1}^{n} \frac{a_{j}}{a_{j}^{0}}\right) p_{n}(x ; \mu),
$$

then $\hat{p}_{n}(x ; \mu)$ and $p_{n}\left(x ; \mu_{0}\right)$ have the same leading coefficient and $\hat{p}_{n}(x ; \mu)$ satisfies the recurrence relation

$$
x \hat{p}_{k}(x ; \mu)=a_{k+1}^{0} \hat{p}_{k+1}(x ; \mu)+b_{k} \hat{p}_{k}(x ; \mu)+\frac{a_{k}^{2}}{a_{k}^{0}} \hat{p}_{k-1}(x ; \mu) .
$$

A linear recurrence relation of second order cannot have three linearly independent solutions; therefore there exist $A_{k}$ and $B_{k}$-independent of $n$-such that

$$
p_{n-k-2}^{(k+2)}\left(x ; \mu_{0}\right)=A_{k} p_{n-k-1}^{(k+1)}\left(x ; \mu_{0}\right)+B_{k} p_{n-k}^{(k)}\left(x ; \mu_{0}\right) .
$$

Setting $n=k$ and $n=k+1$ gives $B_{k}=-a_{k+2}^{0} / a_{k+1}^{0}$ and $A_{k}=$ $a_{k+2}^{0} /\left(a_{k+1}^{0}\right)^{2}\left(x-b_{k}^{0}\right)$, so that

$$
\begin{aligned}
x p_{n-k-1}^{(k+1)}\left(x ; \mu_{0}\right)= & a_{k+1}^{0} p_{n-k}^{(k)}\left(x ; \mu_{0}\right)+b_{k}^{0} p_{n-k-1}^{(k+1)}\left(x ; \mu_{0}\right) \\
& +\frac{\left(a_{k+1}^{0}\right)^{2}}{a_{k+2}^{0}} p_{n-k-2}^{(k+2)}\left(x ; \mu_{0}\right) .
\end{aligned}
$$

(Equation (2.3) can also be derived from (1.5) by using the recurrence formula for $p_{k-1}\left(t ; \mu_{0}\right)$ in the integrand.) Multiply (2.2) by $p_{n-k-1}^{(k+1)}\left(x ; \mu_{0}\right)$ and $(2.3)$ by $\hat{p}_{k}(x ; \mu)$, then subtract the obtained equa- 
tions to find

$$
\begin{aligned}
0= & a_{k+1}^{0}\left(p_{n-k}^{(k)}\left(x ; \mu_{0}\right) \hat{p}_{k}(x ; \mu)-p_{n-k-1}^{(k+1)}\left(x ; \mu_{0}\right) \hat{p}_{k+1}(x ; \mu)\right) \\
& +\left(b_{k}^{0}-b_{k}\right) p_{n-k-1}^{(k+1)}\left(x ; \mu_{0}\right) \hat{p}_{k}(x ; \mu) \\
& +\frac{\left(a_{k+1}^{0}\right)^{2}}{a_{k+2}^{0}} p_{n-k-2}^{(k+2)}\left(x ; \mu_{0}\right) \hat{p}_{k}(x ; \mu) \\
& -\frac{\left(a_{k}\right)^{2}}{a_{k}^{0}} p_{n-k-1}^{(k+1)}\left(x ; \mu_{0}\right) \hat{p}_{k-1}(x ; \mu) .
\end{aligned}
$$

Divide every term by $a_{k+1}^{0}$ and sum from $k=0$ to $k=n-1$. Then one has

$$
\begin{aligned}
\hat{p}_{n}(x ; \mu)= & p_{n}\left(x ; \mu_{0}\right) \\
& +\sum_{k=0}^{n-1}\left\{\frac{b_{k}^{0}-b_{k}}{a_{k+1}^{0}} p_{n-k-1}^{(k+1)}\left(x ; \mu_{0}\right)\right. \\
& \left.\quad+\frac{\left(a_{k+1}^{0}\right)^{2}-\left(a_{k+1}\right)^{2}}{a_{k+1}^{0} a_{k+2}^{0}} p_{n-k-2}^{(k+2)}\left(x ; \mu_{0}\right)\right\} \hat{p}_{k}(x ; \mu) .
\end{aligned}
$$

This is the comparison equation that plays a fundamental role in our investigation of perturbations of $p_{n}\left(x ; \mu_{0}\right)$. The equation shows that $\hat{p}_{n}(x ; \mu)$ is equal to $p_{n}\left(x ; \mu_{0}\right)$ plus a perturbation. The perturbation contains the differences $a_{k+1}^{0}-a_{k+1}$ and $b_{k}^{0}-b_{k}$ and all the associated polynomials $p_{n-k}^{(k)}\left(x ; \mu_{0}\right)$ of order $k=1,2, \ldots, n+1$. This comparison equation enables us to find bounds for the polynomials $p_{n}(x ; \mu)$ if bounds are known for the comparison system $p_{n}\left(x ; \mu_{0}\right)$. Note that it is sufficient to study the orthogonal polynomials $p_{n}(x)$ and functions of the second kind $q_{n}(x)$ for $x \in \mathbb{C}^{+}=\{x \in \mathbb{C}: \mathfrak{I} x \geq 0\}^{1}$ because $p_{n}(\bar{x})=\overline{p_{n}(x)}$ and $q_{n}(\bar{x})=\overline{q_{n}(x)}$.

Lemma 2. Suppose $K$ is a compact set in $\mathbb{C}^{+}$such that

$$
a_{n}^{0} \mathfrak{I}\left(\frac{q_{n-1}\left(x ; \mu_{0}\right)}{q_{n}\left(x ; \mu_{0}\right)}\right) \geq C
$$

where $0<C<\infty$ is some positive constant. Then

$$
\begin{aligned}
& \left|\hat{p}_{n}(x ; \mu) q_{n}\left(x ; \mu_{0}\right)\right| \\
& \quad \leq \frac{1}{C} \exp \left\{\sum_{k=0}^{n-1}\left(\frac{\left|b_{k}^{0}-b_{k}\right|}{C}+\frac{\left|\left(a_{k+1}^{0}\right)^{2}-\left(a_{k+1}\right)^{2}\right|}{C^{2}}\right)\right\}
\end{aligned}
$$

for every $x \in K$.

${ }^{1} \mathfrak{I} x$ denotes the imaginary part of the complex number $x$. 
Proof. Denote by $\mu_{0}^{(k)}$ the orthogonality measure for the $k$ th associated polynomials; then $p_{n}\left(x ; \mu_{0}^{(k)}\right)=p_{n}^{(k)}\left(x ; \mu_{0}\right)$. The functions of the second kind $q_{n-k}\left(x ; \mu_{0}^{(k)}\right)$ are a solution of the recurrence relation (1.1). By minimality and by checking the initial conditions, one finds

$$
a_{k}^{0} q_{n-k}\left(x ; \mu_{0}^{(k)}\right)=\frac{q_{n}\left(x ; \mu_{0}\right)}{q_{k-1}\left(x ; \mu_{0}\right)} .
$$

By using the Wronskian formula

$$
a_{m}\left[p_{m}(x ; \mu) q_{m-1}(x ; \mu)-p_{m-1}(x ; \mu) q_{m}(x ; \mu)\right]=1
$$

for $m=n-k$ and $\mu=\mu_{0}^{(k)}$ one finds

$$
\begin{aligned}
\left|\frac{a_{k}^{0} q_{k-1}\left(x ; \mu_{0}\right)}{p_{n-k}^{(k)}\left(x ; \mu_{0}\right) q_{n}\left(x ; \mu_{0}\right)}\right| & =\left|\frac{1}{p_{n-k}\left(x ; \mu_{0}^{(k)}\right) q_{n-k}\left(x ; \mu_{0}^{(k)}\right)}\right| \\
& =a_{n}^{0}\left|\frac{q_{n-1}\left(x ; \mu_{0}\right)}{q_{n}\left(x ; \mu_{0}\right)}-\frac{p_{n-k-1}^{(k)}\left(x ; \mu_{0}\right)}{p_{n-k}^{(k)}\left(x ; \mu_{0}\right)}\right| \\
& \geq a_{n}^{0}\left|\mathfrak{I}\left\{\frac{q_{n-1}\left(x ; \mu_{0}\right)}{q_{n}\left(x ; \mu_{0}\right)}-\frac{p_{n-k-1}^{(k)}\left(x ; \mu_{0}\right)}{p_{n-k}^{(k)}\left(x ; \mu_{0}\right)}\right\}\right| .
\end{aligned}
$$

The zeros of $p_{n-k-1}^{(k)}\left(x ; \mu_{0}\right)$ and $p_{n-k}^{(k)}\left(x ; \mu_{0}\right)$ interlace. This immediately implies

$$
\mathfrak{I}\left(\frac{p_{n-k-1}^{(k)}\left(x ; \mu_{0}\right)}{p_{n-k}^{(k)}\left(x ; \mu_{0}\right)}\right) \leq 0, \quad x \in \mathbb{C}^{+} .
$$

From $(2.7)$ it follows that the ratio $q_{n}\left(x ; \mu_{0}\right) / q_{n-1}\left(x ; \mu_{0}\right)$ is the Stieltjes transform of a positive measure, and hence

$$
\mathfrak{I}\left(\frac{q_{n-1}\left(x ; \mu_{0}\right)}{q_{n}\left(x ; \mu_{0}\right)}\right) \geq 0, \quad x \in \mathbb{C}^{+} .
$$

Therefore we have for every $x \in K$

$$
\left|p_{n-k}^{(k)}\left(x ; \mu_{0}\right) q_{n-k}\left(x ; \mu_{0}^{(k)}\right)\right| \leq\left\{a_{n}^{0} \mathfrak{I}\left(\frac{q_{n-1}\left(x ; \mu_{0}\right)}{q_{n}\left(x ; \mu_{0}\right)}\right)\right\}^{-1} \leq \frac{1}{C} .
$$


Now multiply both sides of $(2.4)$ by $q_{n}\left(x ; \mu_{0}\right)$ and use $(2.7)$ to obtain $(2.10) \hat{p}_{n}(x ; \mu) q_{n}\left(x ; \mu_{0}\right)$

$$
\begin{aligned}
& =p_{n}\left(x ; \mu_{0}\right) q_{n}\left(x ; \mu_{0}\right) \\
& +\sum_{k=0}^{n-1}\left\{\left(b_{k}^{0}-b_{k}\right) p_{n-k-1}^{(k+1)}\left(x ; \mu_{0}\right) q_{n-k-1}\left(x ; \mu_{0}^{(k+1)}\right)\right. \\
& +\frac{\left(a_{k+1}^{0}\right)^{2}-\left(a_{k+1}\right)^{2}}{a_{k+1}^{0}} \frac{q_{k+1}\left(x ; \mu_{0}\right)}{q_{k}\left(x ; \mu_{0}\right)} \\
& \left.\quad \times p_{n-k-2}^{(k+2)}\left(x ; \mu_{0}\right) q_{n-k-2}\left(x ; \mu_{0}^{(k+2)}\right)\right\} \\
& \times \hat{p}_{k}(x ; \mu) q_{k}\left(x ; \mu_{0}\right) .
\end{aligned}
$$

By using the bounds (2.5) and (2.9) and

$$
\left|\frac{q_{k+1}\left(x ; \mu_{0}\right)}{q_{k}\left(x ; \mu_{0}\right)}\right| \leq \frac{a_{k+1}^{0}}{C}
$$

one finds

$$
\begin{aligned}
\left|\hat{p}_{n}(x ; \mu) q_{n}\left(x ; \mu_{0}\right)\right| \leq & \frac{1}{C}+\sum_{k=0}^{n-1}\left(\frac{\left|b_{k}^{0}-b_{k}\right|}{C}+\frac{\left|\left(a_{k+1}^{0}\right)^{2}-\left(a_{k+1}\right)^{2}\right|}{C^{2}}\right) \\
& \times\left|\hat{p}_{k}(x ; \mu) q_{k}\left(x ; \mu_{0}\right)\right| .
\end{aligned}
$$

The upper bound (2.6) then follows by using Gronwall's inequality (see e.g. [28, p. 440]).

The condition (2.5) is a very natural one and is true for a large class of orthogonal polynomials. Note that

$$
\mathfrak{I}\left(\frac{q_{n-1}\left(x ; \mu_{0}\right)}{q_{n}\left(x ; \mu_{0}\right)}\right)=\frac{\overline{q_{n}\left(x ; \mu_{0}\right)} q_{n-1}\left(x ; \mu_{0}\right)-q_{n}\left(x ; \mu_{0}\right) \overline{q_{n-1}\left(x ; \mu_{0}\right)}}{2 i\left|q_{n}\left(x ; \mu_{0}\right)\right|^{2}},
$$

and by combining the recurrence relations for $q_{n}\left(x ; \mu_{0}\right)$ and $\overline{q_{n}\left(x ; \mu_{0}\right)}$ one has

$$
\begin{array}{r}
a_{n+1}^{0}\left\{q_{n+1}\left(x ; \mu_{0}\right) \overline{q_{n}\left(x ; \mu_{0}\right)}-\overline{q_{n+1}\left(x ; \mu_{0}\right)} q_{n}\left(x ; \mu_{0}\right)\right\} \\
=(x-\bar{x})\left|q_{n}\left(x ; \mu_{0}\right)\right|^{2}+a_{n}^{0}\left\{q_{n}\left(x ; \mu_{0}\right) \overline{q_{n-1}\left(x ; \mu_{0}\right)}\right. \\
\left.\quad-\overline{q_{n}\left(x ; \mu_{0}\right)} q_{n-1}\left(x ; \mu_{0}\right)\right\} .
\end{array}
$$

If $x \in \mathbb{C}^{+} \backslash \mathbb{R}$ then iterating up gives

$$
\begin{aligned}
& a_{n}^{0}\left\{q_{n}\left(x ; \mu_{0}\right) \overline{q_{n-1}\left(x ; \mu_{0}\right)}-\overline{q_{n}\left(x ; \mu_{0}\right)} q_{n-1}\left(x ; \mu_{0}\right)\right\} \\
& \quad=(\bar{x}-x) \sum_{k=n}^{\infty}\left|q_{k}\left(x ; \mu_{0}\right)\right|^{2}
\end{aligned}
$$


which shows that $a_{n}^{0} \mathfrak{I}\left\{q_{n-1}\left(x ; \mu_{0}\right) / q_{n}\left(x ; \mu_{0}\right)\right\}$ is bounded from below on every compact set $K$ in $\mathbb{C}^{+} \backslash \mathbb{R}$. If one iterates (2.12) down then

$$
\begin{aligned}
& a_{n}^{0}\left\{q_{n}\left(x ; \mu_{0}\right) \overline{q_{n-1}\left(x ; \mu_{0}\right)}-\overline{q_{n}\left(x ; \mu_{0}\right)} q_{n-1}\left(x ; \mu_{0}\right)\right\} \\
& \quad=(x-\bar{x}) \sum_{k=0}^{n-1}\left|q_{n}\left(x ; \mu_{0}\right)\right|^{2}+\left\{q_{0}\left(x ; \mu_{0}\right)-\overline{q_{0}\left(x ; \mu_{0}\right)}\right\}
\end{aligned}
$$

hence

$$
a_{n}^{0} \mathfrak{I}\left(\frac{q_{n-1}\left(x ; \mu_{0}\right)}{q_{n}\left(x ; \mu_{0}\right)}\right)=-\frac{(\mathfrak{I} x) \sum_{k=0}^{n-1}\left|q_{k}\left(x ; \mu_{0}\right)\right|^{2}+\mathfrak{I} q_{0}\left(x ; \mu_{0}\right)}{\left|q_{n}\left(x ; \mu_{0}\right)\right|^{2}}
$$

For real $x$ the right-hand side of equation (2.15) can only vanish when $I q_{0}\left(x ; \mu_{0}\right)$ vanishes. If $\mu_{0}$ is absolutely continuous on an interval $[a, b]$ and if the weight function $w_{0}$ is of Dini type on $[a, b]$ (see Lemma 1), then $I q_{0}\left(x ; \mu_{0}\right)=-\pi w_{0}(x)$. This means that the conditions of Lemma 2 are satisfied when $\mu_{0}$ is absolutely continuous on $[a, b]$ with a weight function $w_{0}$ of Dini type on $[a, b]$ and with $K$ a compact set of $\mathbb{C}^{+} \backslash\left\{x \in \mathbb{R}: w_{0}(x)=0\right\}$ not including the endpoints $a$ and $b$. Other conditions on the weight functions $w_{0}$ (e.g. $w_{0}$ may have a finite number of (integrable) singularities as is the case for generalized Jacobi weights) also lead to (2.5) for an appropriate choice of the set $K$.

3. Asymptotic results. An important problem is to find which properties of the comparison system $p_{n}\left(x ; \mu_{0}\right) \quad(n=0,1,2, \ldots)$ are transferred to the system $p_{n}(x ; \mu)(n=0,1,2, \ldots)$ when we are dealing with compact perturbations. One such property is the asymptotic behavior of the ratio of two consecutive orthogonal polynomials:

THEOREM 1. Suppose that $p_{n}(x ; \mu)(n=0,1,2, \ldots)$ is a compact perturbation of $p_{n}\left(x ; \mu_{0}\right)(n=0,1,2, \ldots)$ so that $(1.2)$ holds, and assume that the recurrence coefficients $a_{n+1}^{0}(n=0,1,2, \ldots)$ are bounded. Let $S \subset \mathbb{N}$ be an infinite set of positive integers and define $X(S)$ as the closure of the set of all zeros of $p_{n}(x ; \mu)$ and $p_{n}\left(x ; \mu_{0}\right)$ as $n$ runs through $S$, then

$$
\lim _{n \rightarrow \infty, n \in S} a_{n+1}^{0}\left\{\frac{p_{n+1}\left(x ; \mu_{0}\right)}{p_{n}\left(x ; \mu_{0}\right)}-\frac{\hat{p}_{n+1}(x ; \mu)}{\hat{p}_{n}(x ; \mu)}\right\}=0,
$$

uniformly on compact subsets of $\mathbb{C} \backslash X(S)$.

Proof. Multiply $(2.4)$ by $p_{n+1}\left(x ; \mu_{0}\right)$, then change $n$ to $n+1$ in (2.4) and multiply by $p_{n}\left(x ; \mu_{0}\right)$ and subtract the two resulting equa- 
tions to find

$$
\begin{gathered}
\hat{p}_{n+1}(x ; \mu) p_{n}\left(x ; \mu_{0}\right)-\left(\frac{a_{n+1}}{a_{n+1}^{0}}\right)^{2} \hat{p}_{n}(x ; \mu) p_{n+1}\left(x ; \mu_{0}\right) \\
=\sum_{k=0}^{n} \hat{p}_{k}(x ; \mu)\left\{\frac { b _ { k } ^ { 0 } - b _ { k } } { a _ { k + 1 } ^ { 0 } } \left[p_{n-k}^{(k+1)}\left(x ; \mu_{0}\right) p_{n}\left(x ; \mu_{0}\right)\right.\right. \\
\left.-p_{n-k-1}^{(k+1)}\left(x ; \mu_{0}\right) p_{n+1}\left(x ; \mu_{0}\right)\right] \\
+\frac{\left(a_{k+1}^{0}\right)^{2}-\left(a_{k+1}\right)^{2}}{a_{k+1}^{0} a_{k+2}^{0}} \\
\cdot\left[p_{n-k-1}^{(k+2)}\left(x ; \mu_{0}\right) p_{n}\left(x ; \mu_{0}\right)\right. \\
\left.\left.-p_{n-k-2}^{(k+2)}\left(x ; \mu_{0}\right) p_{n+1}\left(x ; \mu_{0}\right)\right]\right\} .
\end{gathered}
$$

Recall that $p_{n-k-1}^{(k+1)}\left(x ; \mu_{0}\right)$ (for fixed $k$ ) and $p_{n}\left(x ; \mu_{0}\right)$ are two solutions of the recurrence relation (1.1). Their Wronskian is given by

$$
\begin{aligned}
& a_{n+1}^{0}\left\{p_{n-k}^{(k+1)}\left(x ; \mu_{0}\right) p_{n}\left(x ; \mu_{0}\right)-p_{n-k-1}^{(k+1)}\left(x ; \mu_{0}\right) p_{n+1}\left(x ; \mu_{0}\right)\right\} \\
& \quad=a_{k+1}^{0} p_{k}\left(x ; \mu_{0}\right) .
\end{aligned}
$$

If one uses this Wronskian formula in the previous equation, then

$$
\text { (3.3) } \begin{array}{r}
a_{n+1}^{0}\left\{\hat{p}_{n+1}(x ; \mu) p_{n}\left(x ; \mu_{0}\right)-\left(\frac{a_{n+1}}{a_{n+1}^{0}}\right)^{2} \hat{p}_{n}(x ; \mu) p_{n+1}\left(x ; \mu_{0}\right)\right\} \\
=\sum_{k=0}^{n}\left\{\left(b_{k}^{0}-b_{k}\right) p_{k}\left(x ; \mu_{0}\right)\right. \\
\left.+\frac{\left(a_{k+1}^{0}\right)^{2}-\left(a_{k+1}\right)^{2}}{a_{k+1}^{0}} p_{k+1}\left(x ; \mu_{0}\right)\right\} \hat{p}_{k}(x ; \mu) .
\end{array}
$$

Divide by $\hat{p}_{n}(x ; \mu) p_{n}\left(x ; \mu_{0}\right)$. Then

$$
\begin{aligned}
a_{n+1}^{0}\left\{\frac{\hat{p}_{n+1}(x ; \mu)}{\hat{p}_{n}(x ; \mu)}-\left(\frac{a_{n+1}}{a_{n+1}^{0}}\right)^{2} \frac{p_{n+1}\left(x ; \mu_{0}\right)}{p_{n}\left(x ; \mu_{0}\right)}\right\} \\
=\frac{1}{\hat{p}_{n}(x ; \mu) p_{n}\left(x ; \mu_{0}\right)}\left(\sum_{k=0}^{n} c_{k} p_{k}\left(x ; \mu_{0}\right) \hat{p}_{k}(x ; \mu)\right. \\
\left.+\sum_{k=0}^{n} d_{k} p_{k+1}\left(x ; \mu_{0}\right) \hat{p}_{k}(x ; \mu)\right)
\end{aligned}
$$


where

$$
c_{k}=b_{k}^{0}-b_{k}, \quad d_{k}=\frac{\left(a_{k+1}^{0}\right)^{2}-\left(a_{k+1}\right)^{2}}{a_{k+1}^{0}} .
$$

Notice that $c_{k} \rightarrow 0$ and $d_{k} \rightarrow 0$ as $k \rightarrow \infty$. We show that

$$
\lim _{n \rightarrow \infty, n \in S} \frac{1}{\hat{p}_{n}(x ; \mu) p_{n}\left(x ; \mu_{0}\right)} \sum_{k=0}^{n} c_{k} p_{k}\left(x ; \mu_{0}\right) \hat{p}_{k}(x ; \mu)=0
$$

uniformly on compact subsets of $\mathbb{C} \backslash X(S)$; a similar reasoning holds for the sum involving $d_{k}$. By Schwarz' inequality we have

$$
\left|\sum_{k=0}^{n} c_{k} p_{k}\left(x ; \mu_{0}\right) \hat{p}_{k}(x ; \mu)\right|^{2} \leq \sum_{k=0}^{n}\left|c_{k}\right|\left|p_{k}\left(x ; \mu_{0}\right)\right|^{2} \sum_{k=0}^{n}\left|c_{k}\right|\left|\hat{p}_{k}(x ; \mu)\right|^{2}
$$

hence it is sufficient to prove that

$$
\lim _{n \rightarrow \infty, n \in S} \frac{1}{\left|p_{n}\left(x ; \mu_{0}\right)\right|^{2}} \sum_{k=0}^{n-1}\left|c_{k}\right|\left|p_{k}\left(x ; \mu_{0}\right)\right|^{2}=0,
$$

and the same with $\hat{p}_{k}(x ; \mu)$ instead of $p_{k}\left(x ; \mu_{0}\right)$. By standard techniques (3.5) follows if we can show that there is a constant $C$ such that

$$
\frac{1}{\left|p_{n}\left(x ; \mu_{0}\right)\right|^{2}} \sum_{k=0}^{n-1}\left|p_{k}\left(x ; \mu_{0}\right)\right|^{2} \leq C,
$$

uniformly on compact sets of $\mathbb{C} \backslash X(S)$. Let $K$ be a compact set in $\mathbb{C} \backslash X(\mathbb{N})$, then the distance from $K$ to $X(\mathbb{N})$ is strictly positive and we denote this distance by $\delta$. Recall the partial fractions decomposition

$$
\frac{p_{n-1}\left(x ; \mu_{0}\right)}{p_{n}\left(x ; \mu_{0}\right)}=a_{n}^{0} \sum_{j=1}^{n} \frac{\lambda_{j, n} p_{n-1}^{2}\left(x_{j, n} ; \mu_{0}\right)}{x-x_{j, n}}
$$

where $\lambda_{j, n}(1 \leq j \leq n)$ are the Christoffel numbers and $x_{j, n} \quad(1 \leq$ $j \leq n$ ) are the zeros of $p_{n}\left(x ; \mu_{0}\right)$ (see e.g. [26, Theorem 3.3.5], [27, p. 9]). All the zeros of $p_{n}\left(x ; \mu_{0}\right) \quad(n=1,2, \ldots)$ are in $X(\mathbb{N})$; hence $\left|x-x_{j, n}\right| \geq \delta$ and therefore

$$
\left|\frac{p_{n-1}\left(x ; \mu_{0}\right)}{p_{n}\left(x ; \mu_{0}\right)}\right| \leq \frac{a_{n}^{0}}{\delta} \leq \frac{M}{\delta},
$$

where $M$ is a constant such that $a_{n}^{0} \leq M$ for all $n$. From this one finds

$$
\left|\frac{p_{k}\left(x ; \mu_{0}\right)}{p_{n}\left(x ; \mu_{0}\right)}\right| \leq(M / \delta)^{n-k}
$$


and thus

$$
\frac{1}{\left|p_{n}\left(x ; \mu_{0}\right)\right|^{2}} \sum_{k=0}^{n-1}\left|p_{k}\left(x ; \mu_{0}\right)\right|^{2} \leq \sum_{k=0}^{n-1}(M / \delta)^{n-k} \text {. }
$$

If $\delta>M$ then the geometric series on the right-hand side converges and hence (3.6) is true for $\delta$ large enough. Therefore we have shown that the theorem is true on compact sets $K$ which are far enough away from $X(\mathbb{N})$. The left-hand side of (3.1) is uniformly bounded on compact sets of $\mathbb{C} \backslash X(S)$ (this follows easily from (3.7)), and since the full sequence converges to zero on a set with an accumulation point, we can use the Stieltjes-Vitali theorem to complete our proof.

In view of the upper bound (2.6) it is natural to suppose that

$$
\sum_{n=0}^{\infty}\left(\left|b_{n}^{0}-b_{n}\right|+\left|\left(a_{n+1}^{0}\right)^{2}-\left(a_{n+1}\right)^{2}\right|\right)<\infty .
$$

Let us introduce the function

$$
\text { (3.9) } \begin{aligned}
\phi(x)=1+\sum_{k=0}^{\infty}\left\{\left(b_{k}^{0}-b_{k}\right) q_{k}\left(x ; \mu_{0}\right)\right. \\
\left.+\frac{\left(a_{k+1}^{0}\right)^{2}-\left(a_{k+1}\right)^{2}}{a_{k+1}^{0}} q_{k+1}\left(x ; \mu_{0}\right)\right\} \hat{p}_{k}(x ; \mu) .
\end{aligned}
$$

If $K$ is a compact set of $\mathbb{C}^{+}$such that (2.5) holds, then from Lemma $2,(3.8)$ and (2.11) we deduce that $\phi$ is a continuous function in $K$ which is analytic in the interior of $K$. In particular one has

$$
\lim _{y \rightarrow 0+} \phi(x+i y)=\phi(x),
$$

for every $x \in K \cap \mathbb{R}$.

THEOREM 2. Suppose that (3.8) holds and that $K$ is a compact set in $\mathbb{C}^{+}$such that (2.5) holds for every $x \in K$; then

(3.10) $\lim _{n \rightarrow \infty} a_{n+1}^{0}\left\{\hat{p}_{n+1}(x ; \mu) q_{n}\left(x ; \mu_{0}\right)\right.$

$$
\left.-\left(\frac{a_{n+1}}{a_{n+1}^{0}}\right)^{2} \hat{p}_{n}(x ; \mu) q_{n+1}\left(x ; \mu_{0}\right)\right\}=\phi(x)
$$

holds uniformly on $K$. 
Proof. First multiply $(2.4)$ by $q_{n+1}\left(x ; \mu_{0}\right)$, then change $n$ to $n+1$ in (2.4) and multiply by $q_{n}\left(x ; \mu_{0}\right)$. Subtract the two resulting equations and use the Wronskian

$$
\begin{gathered}
\text { (3.11) } a_{n+1}^{0}\left\{p_{n-k}^{(k+1)}\left(x ; \mu_{0}\right) q_{n}\left(x ; \mu_{0}\right)-p_{n-k-1}^{(k+1)}\left(x ; \mu_{0}\right) q_{n+1}\left(x ; \mu_{0}\right)\right\} \\
=a_{k+1}^{0} q_{k}\left(x ; \mu_{0}\right)
\end{gathered}
$$

to find

$$
\begin{array}{r}
a_{n+1}^{0}\left\{\hat{p}_{n+1}(x ; \mu) q_{n}\left(x ; \mu_{0}\right)-\left(\frac{a_{n+1}}{a_{n+1}^{0}}\right)^{2} \hat{p}_{n}(x ; \mu) q_{n+1}\left(x ; \mu_{0}\right)\right\} \\
=1+\sum_{k=0}^{n}\left\{\left(b_{k}^{0}-b_{k}\right) q_{k}\left(x ; \mu_{0}\right)\right. \\
\left.\quad+\frac{\left(a_{k+1}^{0}\right)^{2}-\left(a_{k+1}\right)^{2}}{a_{k+1}^{0}} q_{k+1}\left(x ; \mu_{0}\right)\right\} \hat{p}_{k}(x ; \mu) .
\end{array}
$$

The result then follows immediately.

COROllary 1. Suppose that (3.8) holds. Let $S$ be an infinite set of positive integers and define $X(S)$ as the closure of the zeros of $p_{n}(x ; \mu)$ and $p_{n}\left(x ; \mu_{0}\right)$ as $n$ runs through $S$. Then

$$
\lim _{n \rightarrow \infty, n \in S} \frac{\hat{p}_{n}(x ; \mu)}{p_{n}\left(x ; \mu_{0}\right)}=\phi(x)
$$

uniformly for $x$ on compact subsets of $\mathbb{C}^{+} \backslash X(S)$.

Proof. The left-hand side of (3.10) can be written as

$$
\begin{aligned}
a_{n+1}^{0}\left\{\hat{p}_{n+1}(x ; \mu) q_{n}\left(x ; \mu_{0}\right)-\left(\frac{a_{n+1}}{a_{n+1}^{0}}\right)^{2} \hat{p}_{n}(x ; \mu) q_{n+1}\left(x ; \mu_{0}\right)\right. \\
\cdot \\
=a_{n+1}^{0} \frac{\hat{p}_{n}(x ; \mu)}{p_{n}\left(x ; \mu_{0}\right)}\left\{\frac{\hat{p}_{n+1}(x ; \mu)}{\hat{p}_{n}(x ; \mu)} p_{n}\left(x ; \mu_{0}\right) q_{n}\left(x ; \mu_{0}\right)\right. \\
\left.-\left(\frac{a_{n+1}}{a_{n+1}^{0}}\right)^{2} p_{n}\left(x ; \mu_{0}\right) q_{n+1}\left(x ; \mu_{0}\right)\right\} .
\end{aligned}
$$

The result follows from (3.11) (with $k=-1$ ), Lemma 2 and Theorem 1. 
Corollary 2. Suppose that (3.8) holds and that $\mu_{0}$ is absolutely continuous on $[a, b]$ with a weight function $w_{0}$ which is of Dini type on $[a, b]$. Let $K$ be a closed interval in $[a, b]$ such that $(2.5)$ is satisfied; then

$$
\begin{aligned}
\pi\left(\frac{a_{n+1}}{a_{n+1}^{0}}\right)^{2} \hat{p}_{n}(x ; \mu) \sqrt{w_{0}(x)} \\
\quad-\frac{\left|q_{n}\left(x ; \mu_{0}\right) \phi(x)\right|}{\sqrt{w_{0}(x)}} \sin \left(\arg \phi(x)-\arg q_{n}\left(x ; \mu_{0}\right)\right) \\
\quad=O\left(\sum_{k=n+1}^{\infty}\left(\left|b_{k}^{0}-b_{k}\right|+\left|\left(a_{k+1}^{0}\right)^{2}-\left(a_{k+1}\right)^{2}\right|\right)\right)
\end{aligned}
$$

holds uniformly in $K$.

Proof. Multiply both sides of $(3.12)$ by $\overline{q_{n}\left(x ; \mu_{0}\right)}$ and then take the imaginary part of both sides of the equation to find

$$
\begin{aligned}
& -a_{n+1}^{0}\left(\frac{a_{n+1}}{a_{n+1}^{0}}\right)^{2} \hat{p}_{n}(x ; \mu) \mathfrak{I}\left\{q_{n+1}\left(x ; \mu_{0}\right) \overline{q_{n}\left(x ; \mu_{0}\right)}\right\} \\
& \quad=\mathfrak{I}\left\{\overline{q_{n}\left(x ; \mu_{0}\right)} \phi(x)\right\}-R_{n}(x)
\end{aligned}
$$

where

$$
\begin{aligned}
R_{n}=\mathfrak{I}\left\{\overline{q_{n}\left(x ; \mu_{0}\right)} \sum_{k=n+1}^{\infty}\right. & \left(\left(b_{k}^{0}-b_{k}\right) q_{k}\left(x ; \mu_{0}\right)\right. \\
& \left.\left.+\frac{\left(a_{k+1}^{0}\right)^{2}-\left(a_{k+1}\right)^{2}}{a_{k+1}^{0}} q_{k+1}\left(x ; \mu_{0}\right)\right) \hat{p}_{k}(x ; \mu)\right\} .
\end{aligned}
$$

The remainder $R_{n}(x)$ can easily be estimated by using Lemma 2 . Recall that

$$
a_{n+1}^{0} \mathfrak{I}\left\{q_{n+1}\left(x ; \mu_{0}\right) \overline{q_{n}\left(x ; \mu_{0}\right)}\right\}=\Im q_{0}(x)=-\pi w_{0}(x),
$$

and by $(2.15)$

$$
a_{n}^{0} \mathfrak{I}\left(\frac{q_{n-1}\left(x ; \mu_{0}\right)}{q_{n}\left(x ; \mu_{0}\right)}\right)=\frac{\pi w_{0}(x)}{\left|q_{n}\left(x ; \mu_{0}\right)\right|^{2}} \geq C ;
$$

hence $\left|q_{n}\left(x ; \mu_{0}\right)\right|^{2} \leq \pi w_{0}(x) / C$, and the result follows.

4. The orthogonality measure. In this section we will show that the function $\phi(x)$ defined in (3.9) contains a lot of information concerning the orthogonality measure $\mu$. 
LEMMA 3. Suppose (3.8) holds and that $K$ is a compact set in $\operatorname{supp}\left(\mu_{0}\right)$ such that $(2.5)$ is true. Then $\phi(x) \neq 0$ for all $x \in K$.

Proof. Define

$\psi_{n}(x)=a_{n+1}^{0}\left\{\hat{p}_{n+1}(x ; \mu) q_{n}\left(x ; \mu_{0}\right)-\left(\frac{a_{n+1}}{a_{n+1}^{0}}\right)^{2} \hat{p}_{n}(x ; \mu) q_{n+1}\left(x ; \mu_{0}\right)\right\}$

Then by (3.12) we have

(4.1) $\psi_{n}(x)-\psi_{n-1}(x)=\left\{\left(b_{n}^{0}-b_{n}\right) q_{n}\left(x ; \mu_{0}\right)\right.$

$$
\left.+\frac{\left(a_{n+1}^{0}\right)^{2}-\left(a_{n+1}\right)^{2}}{a_{n+1}^{0}} q_{n+1}\left(x ; \mu_{0}\right)\right\} \hat{p}_{n}(x ; \mu) .
$$

From $(2.5)$ it follows that $q_{n}(x) \neq 0$ for $x \in K$. One easily finds the bounds

$$
\begin{aligned}
\left|\frac{\psi_{n}(x)}{q_{n}\left(x ; \mu_{0}\right)}\right| & \geq\left|\mathfrak{I} \frac{\psi_{n}(x)}{q_{n}\left(x ; \mu_{0}\right)}\right| \\
& =a_{n+1}^{0}\left(\frac{a_{n+1}}{a_{n+1}^{0}}\right)^{2}\left|\hat{p}_{n}(x ; \mu)\right|\left|\mathfrak{I} \frac{q_{n+1}\left(x ; \mu_{0}\right)}{q_{n}\left(x ; \mu_{0}\right)}\right| \\
& =a_{n}^{0}\left(\frac{a_{n+1}}{a_{n+1}^{0}}\right)^{2}\left|\hat{p}_{n}(x ; \mu)\right|\left|\mathfrak{I} \frac{q_{n-1}\left(x ; \mu_{0}\right)}{q_{n}\left(x ; \mu_{0}\right)}\right| \\
& \geq C\left(\frac{a_{n+1}}{a_{n+1}^{0}}\right)^{2}\left|\hat{p}_{n}(x ; \mu)\right|
\end{aligned}
$$

where we have used the equality

$$
a_{n+1}^{0} \mathfrak{I}\left(\frac{q_{n+1}\left(x ; \mu_{0}\right)}{q_{n}\left(x ; \mu_{0}\right)}\right)=-a_{n}^{0} \mathfrak{I}\left(\frac{q_{n-1}\left(x ; \mu_{0}\right)}{q_{n}\left(x ; \mu_{0}\right)}\right) .
$$

By using the bound (4.2) in (4.1) and by the bound

$$
\left|\frac{q_{n+1}\left(x ; \mu_{0}\right)}{q_{n}\left(x ; \mu_{0}\right)}\right| \leq \frac{a_{n+1}^{0}}{C}
$$


one obtains

(4.3) $\left|\psi_{n-1}(x)\right|$

$$
\begin{aligned}
& \leq\left|\psi_{n}(x)\right|\left\{1+\left(\left|b_{n}^{0}-b_{n}\right|\right.\right. \\
& \left.\left.\quad+\frac{\left|\left(a_{n+1}^{0}\right)^{2}-\left(a_{n+1}\right)^{2}\right|}{C}\right) \frac{1}{C a_{n}^{0}}\left(\frac{a_{n+1}^{0}}{a_{n+1}}\right)^{2}\right\} \\
& \leq\left|\psi_{n}(x)\right| \exp \left\{\left(\left|b_{n}^{0}-b_{n}\right|\right.\right. \\
& \left.\left.\quad+\frac{\left|\left(a_{n+1}^{0}\right)^{2}-\left(a_{n+1}\right)^{2}\right|}{C}\right) \frac{1}{C a_{n}^{0}}\left(\frac{a_{n+1}^{0}}{a_{n+1}}\right)^{2}\right\} \\
& \leq\left|\psi_{m}(x)\right| \exp \sum_{k=n}^{m}\left(\left|b_{k}^{0}-b_{k}\right|\right. \\
& \left.\quad+\frac{\left|\left(a_{k+1}^{0}\right)^{2}-\left(a_{k+1}\right)^{2}\right|}{C}\right) \frac{1}{C a_{k}^{0}}\left(\frac{a_{k+1}^{0}}{a_{k+1}}\right)^{2} .
\end{aligned}
$$

which holds for every $m \geq n$. Let $m \rightarrow \infty$; then the sum on the right-hand side converges and $\psi_{m}(x) \rightarrow \phi(x)$ by Theorem 2 . Hence if $\phi(x)=0$ then $\psi_{n}(x)=0$ for infinitely many $n$. But $\psi_{n}(x)=0$ if and only if both $p_{n}(x ; \mu)$ and $p_{n+1}(x ; \mu)$ vanish simultaneously, which is impossible because of the interlacing property of the zeros of orthogonal polynomials.

THEOREM 3. Suppose that $K$ is a compact set in $\operatorname{supp}\left(\mu_{0}\right)$ such that (2.5) holds and assume moreover that (3.8) is true. Then $\mu$ is absolutely continuous with respect to $\mu_{0}$ on $K$ and there exists a version of the Radon-Nikodym derivative $d \mu(x) / d \mu_{0}(x)$ which is continuous on $K$. One has the explicit formula

$$
|\phi(x)|^{2}=\frac{d \mu_{0}(x)}{d \mu(x)}, \quad x \in K
$$

Proof. Consider the Jacobi matrices

$$
\begin{aligned}
J & =\left(\int x p_{i}(x ; \mu) p_{j}(x ; \mu) d \mu(x)\right)_{i, j=0,1,2, \ldots}, \\
J_{0} & =\left(\int x p_{i}\left(x ; \mu_{0}\right) p_{j}\left(x ; \mu_{0}\right) d \mu_{0}(x)\right)_{i, j=0,1,2, \ldots},
\end{aligned}
$$


then $J-J_{0}: l_{2} \rightarrow l_{2}$ is a compact operator. If $\pi(x)$ is a polynomial, then

$$
\pi(J)=\left(\int \pi(x) p_{i}(x ; \mu) p_{j}(x ; \mu) d \mu(x)\right)_{i, j=0,1,2, \ldots}
$$

and similarly for $\pi\left(J_{0}\right)$. From

$$
J^{m}-J_{0}^{m}=\sum_{k=0}^{m-1} J^{k}\left(J-J_{0}\right) J_{0}^{m-k-1}
$$

it follows that $\pi(J)-\pi\left(J_{0}\right)$ is a compact operator for every polynomial $\pi(x)$ (see Kato [18, p. 158]). Now both $\pi(J)$ and $\pi\left(J_{0}\right)$ are banded matrices; hence compactness implies

$$
\begin{aligned}
\lim _{n \rightarrow \infty} \mid \int \pi(x) p_{n}(x ; \mu) p_{n+k}( & x ; \mu) d \mu(x) \\
& -\int \pi(x) p_{n}\left(x ; \mu_{0}\right) p_{n+k}\left(x ; \mu_{0}\right) d \mu_{0} \mid=0,
\end{aligned}
$$

for every integer $k \geq 0$. Introduce the bounded linear functionals

$$
\begin{aligned}
& \Lambda_{n} f=\int_{K} f(x) p_{n}(x ; \mu) p_{n+k}(x ; \mu) d \mu(x), \\
& \Lambda_{n}^{0} f=\int_{K} f(x) p_{n}\left(x ; \mu_{0}\right) p_{n+k}\left(x ; \mu_{0}\right) d \mu_{0}(x),
\end{aligned}
$$

where $f \in C_{K}$, the Banach space of continuous functions on $K$ equipped with the maximum norm, then it follows from the BanachSteinhaus theorem that $\left\|\Lambda_{n}-\Lambda_{n}^{0}\right\|_{C_{K}^{\prime}} \rightarrow 0$ as $n \rightarrow \infty$, where $\|\cdot\|_{C_{K}^{\prime}}$ is the operator norm on $C_{K}^{\prime}$ associated with the maximum norm $\|\cdot\|_{\infty}$ on $C_{K}$. From (2.5) we have

$$
C \leq a_{n}^{0} \mathfrak{I}\left(\frac{q_{n-1}\left(x ; \mu_{0}\right)}{q_{n}\left(x ; \mu_{0}\right)}\right)=\frac{-\mathfrak{I} q_{0}\left(x ; \mu_{0}\right)}{\left|q_{n}\left(x ; \mu_{0}\right)\right|^{2}}=\frac{\pi w_{0}(x)}{\left|q_{n}\left(x ; \mu_{0}\right)\right|^{2}},
$$

hence

$$
\begin{aligned}
& \left|\Lambda_{n}\left(f q_{n}\left(x ; \mu_{0}\right) \overline{q_{n+k}\left(x ; \mu_{0}\right)}\right)-\Lambda_{n}^{0}\left(f q_{n}\left(x ; \mu_{0}\right) \overline{q_{n+k}\left(x ; \mu_{0}\right)}\right)\right| \\
& \quad \leq\left\|\Lambda_{n}-\Lambda_{n}^{0}\right\|_{C_{K}^{\prime}}\left\|f w_{0}\right\|_{\infty} / C \\
& \quad \rightarrow 0 \quad(n \rightarrow \infty) .
\end{aligned}
$$

This implies that the difference of

$$
\begin{aligned}
\left(a_{n+1}^{0}\right)^{2} \int_{K} f(x) \mid \hat{p}_{n+1}(x ; \mu) q_{n}\left(x ; \mu_{0}\right) & \\
& -\left.\left(\frac{a_{n+1}}{a_{n+1}^{0}}\right)^{2} \hat{p}_{n}(x ; \mu) q_{n+1}\left(x ; \mu_{0}\right)\right|^{2} d \mu(x)
\end{aligned}
$$


and

$$
\begin{aligned}
& \left(a_{n+1}^{0}\right)^{2} \int_{K} f(x) \mid p_{n+1}\left(x ; \mu_{0}\right) q_{n}\left(x ; \mu_{0}\right) \\
& -\left.p_{n}\left(x ; \mu_{0}\right) q_{n+1}\left(x ; \mu_{0}\right)\right|^{2} d \mu_{0}(x)
\end{aligned}
$$

converges to zero as $n \rightarrow \infty$ whenever $\left\|f w_{0}\right\|_{\infty}$ is finite. From Theorem 2 we have

$$
\begin{aligned}
\lim _{n \rightarrow \infty}\left(a_{n+1}^{0}\right)^{2} \int_{K} f(x) \mid \hat{p}_{n+1}(x ; \mu) q_{n}\left(x ; \mu_{0}\right) \\
\quad-\left.\left(\frac{a_{n+1}}{a_{n+1}^{0}}\right)^{2} \hat{p}_{n}(x ; \mu) q_{n+1}\left(x ; \mu_{0}\right)\right|^{2} d \mu(x) \\
=\int_{K} f(x)|\phi(x)|^{2} d \mu(x),
\end{aligned}
$$

and by the Wronskian formula (3.11) (with $k=-1$ ) one has

$$
\begin{aligned}
& \left(a_{n+1}^{0}\right)^{2} \int_{K} f(x) \mid p_{n+1}\left(x ; \mu_{0}\right) q_{n}\left(x ; \mu_{0}\right) \\
& \quad-\left.p_{n}\left(x ; \mu_{0}\right) q_{n+1}\left(x ; \mu_{0}\right)\right|^{2} d \mu_{0}(x) \\
& \quad=\int_{K} f(x) d \mu_{0}(x) .
\end{aligned}
$$

By comparing the right-hand side of (4.5) and (4.6) we find that $\mu_{0}$ is absolutely continuous with respect to $\mu$ on $K$ and since $\phi(x) \neq 0$ on $K$ (by Lemma 3) it also follows that $\mu$ is absolutely continuous with respect to $\mu_{0}$ on $K$. This proves the theorem.

\section{Examples.}

5.1. Finite perturbations. Suppose that the recurrence coefficients of $p_{n}\left(x ; \mu_{m}\right)$ agree with the recurrence coefficients of $p_{n}\left(x ; \mu_{0}\right)$ from a certain index $m$ onward, i.e.

$$
a_{n+1}=a_{n+1}^{0}, \quad b_{n}=b_{n}^{0} \quad n \geq m .
$$

It is clear that (3.8) is true whenever one deals with these finite perturbations. From (3.9) and (3.12) it follows that the function $\phi(x)=$ $\phi_{m}(x)$ is given by

$$
\begin{aligned}
\phi_{m}(x)=a_{n}^{0}\left\{\hat{p}_{n}\left(x ; \mu_{m}\right) q_{n-1}\left(x ; \mu_{0}\right)\right. & \\
& \left.-\left(\frac{a_{n}}{a_{n}^{0}}\right)^{2} \hat{p}_{n-1}\left(x ; \mu_{m}\right) q_{n}\left(x ; \mu_{0}\right)\right\}, \quad n \geq m .
\end{aligned}
$$


Choose $n=m+1$; then

$$
\begin{aligned}
\left|\phi_{m}(x)\right|^{2}=\left(a_{m+1}^{0}\right)^{2} & \hat{p}_{m+1}^{2}\left(x ; \mu_{m}\right)\left|q_{m}\left(x ; \mu_{0}\right)\right|^{2} \\
& +\hat{p}_{m}^{2}\left(x ; \mu_{m}\right)\left|q_{m+1}\left(x ; \mu_{0}\right)\right|^{2}-2 \hat{p}_{m}\left(x ; \mu_{m}\right) \\
& \left.\cdot \hat{p}_{m+1}\left(x ; \mu_{m}\right) \mathfrak{R}\left[q_{m}\left(x ; \mu_{0}\right) \overline{q_{m+1}\left(x ; \mu_{0}\right)}\right]\right\} .
\end{aligned}
$$

The Radon-Nikodym derivative $d \mu_{0} / d \mu$ can therefore be computed explicitly by using Lemma 1 . This requires the computation of Cauchy principal values and thus one needs a numerical technique that deals with singular integrals of this type (see e.g. [8]).

5.2. Perturbations of Jacobi polynomials. The recurrence coefficients for the orthonormal Jacobi polynomials $p_{n}^{(\alpha, \beta)}(x)$ are given by

$$
\begin{aligned}
\left(a_{n}^{0}\right)^{2} & =\frac{4 n(n+\alpha)(n+\beta)(n+\alpha+\beta)}{(2 n+\alpha+\beta-1)(2 n+\alpha+\beta)^{2}(2 n+\alpha+\beta+1)} \\
& =\frac{1}{4}+\frac{1-2\left(\alpha^{2}+\beta^{2}\right)}{16 n^{2}}+O\left(n^{-3}\right), \\
b_{n}^{0} & =\frac{\beta^{2}-\alpha^{2}}{(2 n+\alpha+\beta)(2 n+\alpha+\beta+2)}=\frac{\beta^{2}-\alpha^{2}}{4 n^{2}}+O\left(n^{-3}\right),
\end{aligned}
$$

where $\alpha, \beta>-1$ and if $\alpha=-\beta$ one has $b_{0}=\beta$. If (3.8) is satisfied, then this means that the recurrence coefficients of $p_{n}(x ; \mu)$ satisfy

$$
\sum_{k=0}^{\infty}\left(\left|1-4 a_{k+1}^{2}\right|+2\left|b_{k}\right|\right)<\infty
$$

but then it is more convenient to use the constant recurrence coefficients $a_{n}^{0}=1 / 2, b_{n}=0$ as comparison system, and such perturbations have been studied quite well ([11], [21], [28]). It would be of interest to find results under the stronger condition

$$
\sum_{n=0}^{\infty} n^{\gamma}\left(\left|b_{n}^{0}-b_{n}\right|+\frac{\left|\left(a_{n+1}^{0}\right)^{2}-\left(a_{n+1}\right)^{2}\right|}{a_{n+1}^{0}}\right)<\infty
$$

with $\gamma>0$, but in order to do this we need bounds of the type

$$
\left|p_{n-k}\left(x ; \mu_{0}^{(k)}\right) q_{n-k}\left(x ; \mu_{0}^{(k)}\right)\right| \leq C(n+1)^{\gamma}, \quad x \in K,
$$

where $\mu_{0}^{(k)}$ is the orthogonality measure for the associated Jacobi polynomials of order $k$. 
THEOREM 4. Suppose that $\left|q_{0}\left(x ; \mu_{0}^{(n+1)}\right)\right| \leq C$ is valid on an interval $K$ of the real line and that (5.5) holds. Then

$$
\begin{aligned}
\left|\hat{p}_{n}(x ; \mu) q_{n}\left(x ; \mu_{0}\right)\right| & \\
\leq C(n+1)^{\gamma} \exp & \left\{\sum_{k=0}^{n-1}(k+1)^{\gamma}\right. \\
& \left.\cdot\left(C\left|b_{k}^{0}-b_{k}\right|+C^{2}\left|\left(a_{k+1}^{0}\right)^{2}-\left(a_{k+1}\right)^{2}\right|\right)\right\} .
\end{aligned}
$$

Moreover the function $\phi$ defined in (3.9) is continuous on $K$ and Theorem 2 (with its corollaries) remains valid.

Proof. The bound follows by inserting the upper bound (5.5) into (2.10) and by using

$$
\frac{q_{k+1}\left(x ; \mu_{0}\right)}{q_{k}\left(x ; \mu_{0}\right)}=a_{k+1}^{0} q_{0}\left(x ; \mu_{0}^{(k+1)}\right) .
$$

One can then repeat the proof of Theorem 2 with these bounds.

5.3. Perturbations of Pollaczek polynomials. The recurrence coefficients for orthonormal Pollaczek polynomials are given by

$$
\begin{aligned}
\left(a_{n}^{0}\right)^{2} & =\frac{n(n+2 \lambda-1)}{4(n+\lambda+a-1)(n+\lambda+a)} \\
& =\frac{1}{4}-\frac{a}{2 n}+O\left(n^{-2}\right), \\
b_{n}^{0} & =\frac{-b}{n+\lambda+a}=-\frac{b}{n}+O\left(n^{-2}\right),
\end{aligned}
$$

where $\lambda>0$ and $a+\lambda>0$ or $-1 / 2<\lambda<0$ and $-1<\lambda+a<0$. It would be of interest to find bounds of the type (5.5) with $K=[-1,1]$, where $\mu_{0}^{(k)}$ is the orthogonality measure for associated Pollaczek polynomials of order $k$, because $[-1,1]$ is the essential spectrum for Pollaczek polynomials.

5.4. Generalized Jacobi weights, sieved weights. A generalized Jacobi weight [3], [21] is of the form

$$
w_{0}(x)=\varphi(x)(1+x)^{\gamma_{1}} \prod_{k=2}^{N-1}\left|t_{k}-x\right|^{\gamma_{k}}(1-x)^{\gamma_{N}}, \quad-1<x<1,
$$


where $\gamma_{k}>-1,-1<x_{2}<x_{3}<\cdots<x_{N-1}<1$ and $\varphi$ is a positive function on $[-1,1]$ of Dini type. The recurrence coefficients of generalized Jacobi polynomials are not known in general, but for some special cases (such as sieved ultraspherical [1] or sieved Jacobi polynomials) explicit formulas are known which show some periodic oscillations. For sieved orthogonal polynomials obtained through a polynomial transformation of an ultraspherical weight, i.e. when considering the weight

$$
\begin{aligned}
w_{0}(x) & =\left|U_{k-1}(x)\right|\left(1-T_{k}^{2}(x)\right)^{\lambda} \\
& =\left(1-x^{2}\right)^{\lambda}\left|U_{k-1}(x)\right|^{2 \lambda+1}, \quad-1<x<1,
\end{aligned}
$$

one has [13]

$$
\begin{aligned}
b_{n} & =0, \quad a_{n k+j}=1 / 2 \quad(j=2, \ldots, k-1), \\
a_{k n}^{2} & =\frac{n(n+2 \lambda)}{2\left[4(n+\lambda)^{2}-1\right]} \frac{c_{n-1}(1)}{c_{n}(1)}, \quad a_{k n+1}^{2}=\frac{1}{2}-a_{k n}^{2} .
\end{aligned}
$$

where $c_{n}(x)$ are the orthonormal ultraspherical polynomials with weight $\left(1-x^{2}\right)^{\lambda}$. This means that when recurrence coefficients oscillate around their limiting values, then the weight may have singularities inside the interval on which orthogonality holds.

\section{REFERENCES}

[1] W. Al-Salam, W. Allaway and R. Askey, Sieved ultraspherical polynomials, Trans. Amer. Math. Soc., 284 (1984), 39-55.

[2] R. Askey and J. Wimp, Associated Laguerre and Hermite polynomials, Proc. Royal Soc. Edinburgh, 96A (1985), 15-37.

[3] V. Badkov, Convergence in mean and almost everywhere of Fourier series in orthogonal polynomials, Mat. Sb., 95 (137) (1974), 229-262.

[4] W. Barrett, An asymptotic formula relating to orthogonal polynomials, J. London Math. Soc., (2) 6 (1973), 701-704.

[5] P. Barrucand and D. Dickinson, On the associated Legendre polynomials, in Orthogonal Expansions and their Continuous Analogs (D. T. Haimo, ed.), Southern Illinois Univ. Press, Edwardsville, 1968, pp. 43-50.

[6] K. M. Case, Orthogonal polynomials from the viewpoint of scattering theory, J. Math. Phys., 15 (1974), 2166-2174.

[7] T. S. Chihara, Orthogonal Polynomials, Gordon and Breach, New York, 1978.

[8] G. Criscuolo and G. Mastroianni, On the convergence of an interpolatory product rule for evaluating Cauchy principal value integrals, Math. Comp., 48 (1987), 725-735. 
[9] J. Dombrowski and P. Nevai, Orthogonal polynomials, measures and recurrence relations, SIAM J. Math. Anal., 17 (1986), 752-759.

[10] J. S. Geronimo, On the spectra of infinite dimensional Jacobi matrices, J. Approx. Theory, 53 (1988), 251-265.

[11] J. S. Geronimo and K. M. Case, Scattering theory and polynomials orthogonal on the real line, Trans. Amer. Math. Soc., 258 (1980), 467-494.

[12] J. S. Geronimo and W. Van Assche, Orthogonal polynomials with asymptotically periodic recurrence coefficients, J. Approx. Theory, 46 (1986), 251-283.

[13] __, Orthogonal polynomials on several intervals via a polynomial mapping, Trans. Amer. Math. Soc., 308 (1988), 559-581.

[14] Ya. L. Geronimus, On some finite difference equations and corresponding systems of orthogonal polynomials, Zap. Mat. Otd. Fiz.-Mat. Fak. i Kharkov. Mat. Obšč., (4) 25 (1957), 87-100 (in Russian).

[15] C. C. Grosjean, The measure induced by orthogonal polynomials satisfying a recursion formula with either constant or periodic coefficients, part I: constant coefficients, Acad. Analecta, Koninkl. Acad. Wetensch. Lett. Sch. Kunsten België, 48 (3) (1986), 39-60.

[16] _ The measure induced by orthogonal polynomials satisfying a recursion formula with either constant or periodic coefficients, part II: pure or mixed periodic coefficients, Acad. Analecta, Koninkl. Acad. Wetensch. Lett. Sch. Kunsten België, 48 (5) (1986), 55-94.

[17] _ The weight functions, generating functions and miscellaneous properties of the sequences of orthogonal polynomials of the second kind associated with the Jacobi and the Gegenbauer polynomials, J. Comput. Appl. Math., 16 (1986), 259-307.

[18] T. Kato, Perturbation Theory for Linear Operators, Springer-Verlag, Berlin, 1966.

[19] A. Máté and P. Nevai, Sublinear perturbations of the differential equation $y^{(n)}=$ 0 and of the analogous difference equation, J. Differential Equations, 53 (1984), 234-257.

[20] A. Máté, P. Nevai and V. Totik, Extensions of Szegö's theory of orthogonal polynomials, II, Constr. Approx., 3 (1987), 51-72.

[21] P. G. Nevai, Orthogonal Polynomials, Memoirs Amer. Math. Soc., vol. 231, Amer. Math. Soc., Providence, R.I., 1979.

[22] _ A new class of orthogonal polynomials, Proc. Amer. Math. Soc., 91 (1984), 409-415.

[23] O. Perron, Die Lehre von den Kettbrüchen, Chelsea Publ. Company, New York, 1950.

[24] J. Sherman, On the numerators of the convergents of the Stieltjes continued function, Trans. Amer. Math. Soc., 35 (1933), 64-87.

[25] T. J. Stieltjes, Recherches sur les fractions continues, Ann. Fac. Sci. Toulouse, 8 (1894), J1-122; 9 (1895), A1-47.

[26] G. Szegö, Orthogonal Polynomials, Amer. Math. Soc. Colloq. Publ., vol. 23, Amer. Math. Soc., Providence, R.I., 4th edition 1975.

[27] W. Van Assche, Asymptotics for Orthogonal Polynomials, Lecture Notes in Mathematics, vol. 1265, Springer-Verlag, Berlin, 1987.

[28] _ Asymptotics for orthogonal polynomials and three-term recurrences, in "Orthogonal Polynomials: Theory and Practice" (P. Nevai, ed.), NATO ASI Series C 294, Kluwer Academic Publishers, Dordrecht, 1990, pp. 435-462.

[29] J. Wimp, Explicit formulas for the associated Jacobi polynomials and some applications, Canad. J. Math., 39 (1987), 983-1000. 
[30] A. Wintner, Spektraltheorie der unendlichen Matrizen, Hirzel, Leipzig, 1929.

[31] A. Zygmund, Trigonometrical Series, Chelsea Publishing Company, New York, 1952.

Received July 27, 1990 and in revised form June 3, 1991. This material is based on research supported by the National Science Foundation under grant DMS- 8814488 and by NATO (first author) and by a Research Grant from the Belgian National Fund for Scientific Research (second author). The second author is a Research Associate of the Belgian National Fund for Scientific Research.

The Ohio State University

Columbus, OH 43210-1174

AND

KATHOLIEKe UNIVERSITEIT LEUVEN

Celestijnenlaan 200B

B-3001 Heverlee (Leuven) Belgium 


\section{PACIFIC JOURNAL OF MATHEMATICS EDITORS}

V. S. VARADARAJAN

(Managing Editor)

University of California

Los Angeles, CA 90024-1555-05

Herbert Clemens

University of Utah

Salt Lake City, UT 84112

F. Michael Christ

University of California

Los Angeles, CA 90024-1555

THOMAS ENRIGHT

University of California, San Diego

La Jolla, CA 92093

\section{C. MOORE}

University of California

Berkeley, CA 94720

MARTIN SCHARLEMANN

University of California

Santa Barbara, CA 93106

HAROLD STARK

University of California, San Diego

La Jolla, CA 92093

STEVEN KERCKHOFF

Stanford University

Stanford, CA 94305

ASSOCIATE EDITORS

R. ARENS

E. F. BECKENBACH (1906-1982)

B. H. NeumanN

F. WOLF

(1904-1989)

K. YosHIDA

SUPPORTING INSTITUTIONS

UNIVERSITY OF ARIZONA

UNIVERSITY OF OREGON

UNIVERSITY OF BRITISH COLUMBIA

UNIVERSITY OF SOUTHERN CALIFORNIA

CALIFORNIA INSTITUTE OF TECHNOLOGY

UNIVERSITY OF CALIFORNIA

MONTANA STATE UNIVERSITY

STANFORD UNIVERSITY

UNIVERSITY OF NEVADA, RENO

UNIVERSITY OF HAWAII

NEW MEXICO STATE UNIVERSITY

OREGON STATE UNIVERSITY

UNIVERSITY OF UTAH

WASHINGTON STATE UNIVERSITY

UNIVERSITY OF WASHINGTON 


\section{Pacific Journal of Mathematics}

\section{Vol. 153, No. $1 \quad$ March, 1992}

Patrick Robert Ahern and Carmen Cascante, Exceptional sets for Poisson integrals of potentials on the unit sphere in $\mathbf{C}^{n}, p \leq 1 \ldots \ldots \ldots \ldots \ldots$

David Peter Blecher, The standard dual of an operator space ........... 15

Patrick Gilmer, Real algebraic curves and link cobordism .............. 31

Simon M. Goberstein, On orthodox semigroups determined by their

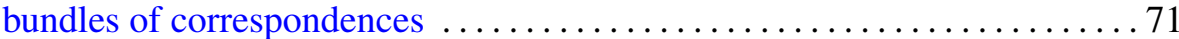

John Kalliongis and Darryl John McCullough, Homeotopy groups of irreducible 3-manifolds which may contain two-sided projective planes .......................................... 85

Yuji Konishi, Masaru Nagisa and Yasuo Watatani, Some remarks on actions of compact matrix quantum groups on $C^{*}$-algebras $\ldots \ldots \ldots \ldots 119$

Guojun Liao and Luen-Fai Tam, On the heat equation for harmonic maps from noncompact manifolds ........................... 129

John Marafino, Boundary behavior of a conformal mapping . .......... 147

Ji Min, A remark on the symmetry of solutions to nonlinear elliptic

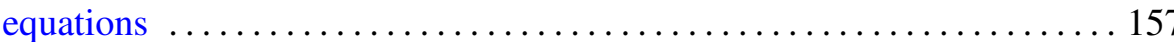

Paul Nevai and Walter Van Assche, Compact perturbations of orthogonal

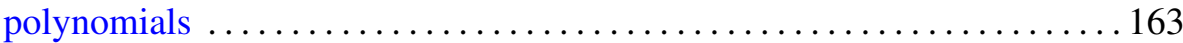

Kyril Tintarev, Level set maxima and quasilinear elliptic problems ....... 185 